1. Young EJ. Brucella species. In: Mandell, Douglas and Bennett's Principles and Practice of Infectious diseases. 4th edition. Churchill Livingstone N York, 1995; 2053-7.

2. García Porrua C, González Gay MA, Ibáñez D, García País MJ. The clinical spectrum of severe septic bursitis in northwestern Spain: a 10 years study. J Rheumatol 1999; 26: 663-7.

3. Brooks GF, Butel JS, Ornston LN. Medical Microbiology. 20th edition. Appleton and Lange, 1995; 235-7.

4. Bercovich Z. Maintenance of Brucella abortus-free herds: a review with emphasis on the epidemiology and the problems in diagnosing brucellosis in areas of low prevalence. Vet Q 1998; 20: 3, 81-8.

5. Ozgül A, Yaziciouglu K, Gündüz S, Kalyon TA, Arpacioglu O. Acute brucella sacroilitis: clinical features. Clin Rheumatol 1998; 17: 6; 521-3.

6. González Gay MA, García Porrua C, Ibáñez D, García País MJ. Osteoarticular complications of brucellosis in an atlantic area of Spain. J Rheumatol 1999; 26:1; 141-5.

7. Solera J, Martínez Alfaro E, Espinosa A. Recognition and optimum treatment of brucellosis. Drugs 1997; 53:2; 245-6.

\section{Metástasis coroideas y cutáneas como forma de presentación de un adenocarcinoma esofágico}

\section{Sr. Director:}

Las neoplasias malignas ocasionalmente metastatizan en estructuras intraoculares. La mayoría de ellas en la uvea (88\% en la coroides). En el $25 \%$ de los casos la lesión intraocular representa la primera manifestación del cáncer sistémico (1). La mama y el pulmón representan más del $80 \%$ del origen del tumor primario. Las metástasis cutáneas son expresión de una enfermedad neoplásica representando el $10 \%$ de la enfermedad metastásica y normalmente se presentan cuando el tumor primitivo está muy avanzado (2). Los tumores de mama, pulmón, colon y el melanoma son los que más frecuentemente metastatizan en piel. Presentamos un paciente con un adenocarcinoma esofágico cuya forma de presentación fue la presencia de metástasis oculares y cutáneas.

Varón de 47 años de edad ex fumador moderado que un mes antes de nuestra valoración presentó de forma brusca dolor y pérdida de visión en ojo izquierdo. La exploración oftalmológica mostró una masa sólida redondeada, con hiperfluorescencia precoz y escasa elevación ocupante de todo el polo posterior y asociada a exudación subrretiniana inferior, sin signos inflamatorios asociados (Fig. 1). 3 semanas antes notó la aparición de bultomas en cuero cabelludo y 15 días antes disfagia para sólidos. Junto a ello refería astenia, anorexia y pérdida de unos $8 \mathrm{Kg}$ de peso. En la exploración presentaba lesiones de vitíligo en manos y 3 nódulos eritematosos, dolorosos a la palpación en cuero cabelludo . En la analítica destacaba una anemia normocítica (Hb: 7,8 gr/dl), una VSG de $62 \mathrm{~mm}$ a la la hora y una GGT y una FA elevadas. La radiografia de tórax mostraba un ensanchamiento del mediastino superior.

En la ecografia ocular se vio una elevación máxima de $3 \mathrm{~mm}$, reflectividad interna media/alta que reafirmaba la impresión diagnóstica inicial de lesión metastásica coroidea. Dos meses después se observaron nuevas lesiones en ojo derecho, múltiples, de idénticas características. La tomografia computariada (TAC) torácica y abdominal mostraba una tumoración esofágica con imágenes compatibles con metástasis ganglionares, pulmonares, hepáticas y óseas. La gammagrafia ósea era compatible con metástasis a nivel de la calota craneal, D12, $10^{\mathrm{a}}$ y $11^{\mathrm{a}}$ costilla derecha y fémur derecho. La endoscopia confirmó la presencia de una tumoración de esófago proximal desde los 20 a los $30 \mathrm{~cm}$ de la arcada dental. Se realizó biopsia informada como adenocarcinoma moderadamente diferenciado infiltrante. La biopsia de la lesión cutánea (cuero cabelludo) era compatible con metástasis de adenocarcinoma

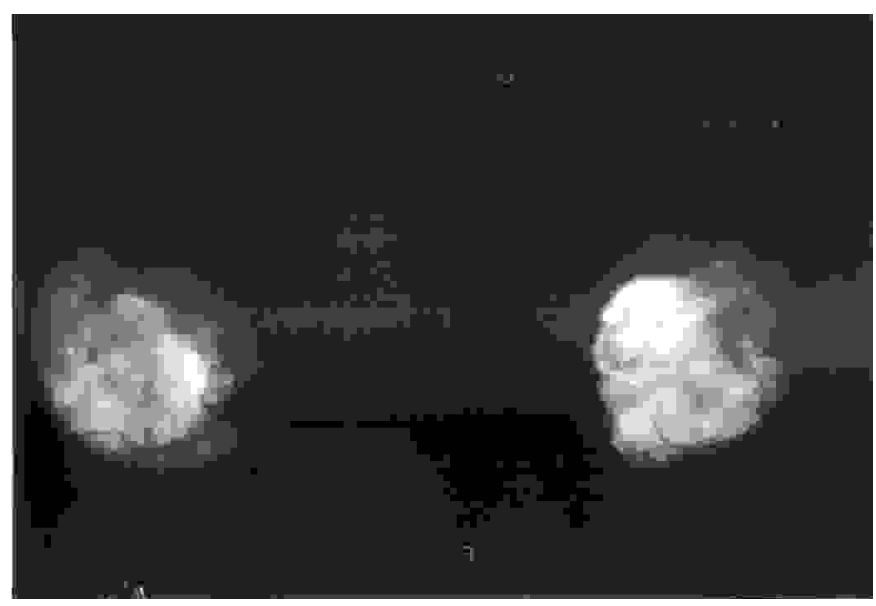

Fig. 1. M asa en el polo posterior del ojo izquierdo.

moderadamente diferenciado. Se indicó exclusivamente tratamiento sintomático con radioterapia paliativa.

La edad media de presentación de las metástasis oculares es de 52 años de edad y sólo en el $10 \%$ de los casos supone la $1^{\text {a }}$ metástasis identificada (3).

El tracto gastrointestinal (colorectales sobre todo) es la localización del tumor primario en el $4 \%$ de los casos (1). En estos casos las metástasis uveales suelen ser unilaterales y de un grosor mayor que en otro tipo de tumores.Las metástasis cutáneas se presentan con 4 patrones metastásicos clásicos: nodular, inflamatorio o infiltrativo, esclerodermiforme y formas atípicas (4). La correlación entre las características histológicas de la lesión primitiva y la metastásica suele ser buena, ofreciendo las lesiones metastásicas un menor grado de diferenciación (5).

En revisión de la literatura (MEDLINE 1966-1999) sólo encontramos una referencia en la que se asocia un carcinoma esofágico con metástasis en iris (6). La asociación de metástasis cutáneas y tumores esofágicos está descrita también de forma excepcional (7-9).

En presencia de metástasis intraoculares y/o cutáneas consideramos que se debe descartar en primer lugar los tumores de mama en la mujer y el pulmón en el varón sin olvidarnos de otras localizaciones como el tracto gastrointestinal (esófago en nuestro caso), riñón, etc.

\section{J. Noval Menéndez, F. J. Nuño Mateo, E. Rodríguez Álva- rez*, P. Pérez Solís**, Y. López Álvarez**}

Servicio de Medicina Interna, *Servicio de Dermatología y **Servicio de Medicina Familiar y Comunitaria. Hospital de Cabueñes. Gijón.

1. Shields CL, Shields JAt Gross NE, Schwartz GP, Lally SE. Survey of 520 eyes with uveal metastases. Ophthalmology 1997; 104: 1265-76.

2. Lookingbill D, Spangler N, Helm KF. Cutaneous metastasis in patients with metastasic carcinoma. A retrospective study of 4020 patients. J Am Acad Dermatol 1993; 29: 228-36.

3. Maor M, Chan RC, Young SE. Radioterapy of choroid metastasis: breast cancer in primary ite. Cancer 1997; 40: 2081-6.

4. Brownstein MH, Helwig EB. Spread of tumours to the skin: Arch Dermatol 1973, 107:80-6.

5. Coslett LM, Katlic MR. Lung cancer with skin metastases. Chest 1990; 97: 756-9.

6. Shields JA, Shields CL, Kiratli H, de Potter P. Metastatic tumors to the iris in 40 patients. Am J Ophthalmol 1995; 119: 422-30. 
7. Farr P, Goens J, Herchuelz J, Van Hasselt C, Litvin B. Cutaneous metastasis as the first manifestation of adenocarcinoma in Barret esophagus. J Belge Radiol 1987; 70: 329-32.

8. Peracchia A, Rosati R, Fumagalli U, Bona S, Chella B. Thoracoscopic disecction of the esophagus for cancer. Int Surg 1997; 82,1: 1-4

9. SibrackLA. Cutaneous signs of internal malignant disease. Prim Care 1978; 5: 263-80.

\section{Fibrilación ventricular durante la administración intravenosa de fenitoína}

\section{Sr. Director:}

La fenitoína es un fármaco de primera línea en el tratamiento de la epilepsia (1-4). Su administración intravenosa puede producir efectos cardiovasculares adversos como hipotensión, bradicardia, alteraciones del ritmo (1-7) y miocarditis por hipersensibilidad (2). La fibrilación ventricular relacionada con la administración de fenitoína es muy rara (8). Presentamos un caso.

Mujer de 37 años con retraso mental y epilepsia en tratamiento con fenitoína $100 \mathrm{mg} / 12$ horas y tioridazina. La familia consultó por síncope y relajación de esfinteres. En urgencias se objetivaron varias crisis epilépticas parciales de miembro superior derecho con posterior generalización. Ingresa en la unidad de cuidados intensivos. Exploración: TA $120 / 60 \mathrm{mmHg}$, frecuencia cardíaca $83 \mathrm{I} / \mathrm{min}$, comatosa, no obedece órdenes, localiza dolor, resistencia a la apertura ocular, mala higiene personal, auscultación cardiopulmonar y abdomen sin alteraciones. Al ingreso hemograma y bioquímica (incluidos calcio y magnesio) normales. Gasometría basal: $\mathrm{pH} 7,49 ; \mathrm{pCO}_{2} 29,5 \mathrm{mmHg} ; \mathrm{PO}_{2} 74$ $\mathrm{mmHg} ; \mathrm{HCO}_{3}$ 22,9. ECG: ritmo sinusal a $70 \mathrm{I} / \mathrm{min}$; QTc 0,32 segundos; alteraciones difusas de la repolarización. Radiografía tórax: normal. Se administra $1 \mathrm{gr}$ de fenitoína en $500 \mathrm{ml}$ de suero salino a $8 \mathrm{mg} / \mathrm{min}$, cediendo las crisis epilépticas. Cuando se habían infundido $900 \mathrm{mg}$ de fenitoína, se evidenciaba fibrilación ventricular (Fig. 1a) que revertía a ritmo sinusal tras choque eléctrico de 200 julios. Se suspendía la fenitoína, pese a ello presentaba extrasistolia ventricular multifocal frecuente y cinco episodios de fibrilación-flutter ventricular (Fig. 1b) en las siguientes 4 horas. Los niveles de fenitoína tras suspender su perfusión eran $11,4 \mathrm{llg} / \mathrm{ml}$. La paciente fue dada de alta hospitalaria con fenitoína $100 \mathrm{mg} / 12 \mathrm{~h}$ y topiramato $25 \mathrm{mg} / 12 \mathrm{~h}$.

La administración intravenosa de fenitoína es efectiva en las crisis epilépticas repetidas, deteniendo el status epiléptico en más del $50 \%$ de casos, siendo su tratamiento de elección combinada con benzodiacepinas $(3,5,7)$. También es un antiarrítmico clase $\mathrm{IB}$, ha sido usada en el tratamiento de arritmias ventriculares tras infarto miocárdico, fibrilación auricular paroxística y arritmias ventriculares causadas por digital $(1,6,8-10)$.

La toxicidad de la fenitoína depende de la vía de administración, la duración de la exposición y dosis (2-5,7). La seguridad de la fenitoína intravenosa ha sido cuestionada por dos razones. Primero, su administración se ha asociado con graves complicaciones como parada cardiorrespiratoria $(1,3,6)$. Segundo, la fenitoína es muy liposoluble, pero poco hidrosoluble $(4,7)$. Esto último condiciona, por un lado que la fenitoína sea muy inestable en muchos diluyentes (dextrosa, salino normal o Ringer lactato) pudiendo cristalizar y precipitar $(4,7)$; además la solución intavenoa contiene propilen glicol, etanol e hidróxido sódico $(2,3,7)$. La fenitoína intravenosa se asocia con un $25 \%$ de complicaciones (3), que pueden dividirse en locales y sistémicas. Las primeras son dolor, flebitis y daño tisular en el sitio de infusión; son las más frecuentes y pueden disminuirse con el uso de soluciones menos concentradas $(<6,7 \mathrm{mg} / \mathrm{ml})$ y catéteres adecuados. Las complicaciones sistémicas pueden ser neurológicas (ataxia, confusión, diplopía, vértigo, etc.), cardiovasculares y gastrointestinales (náusea, vómitos) $(3,4)$.

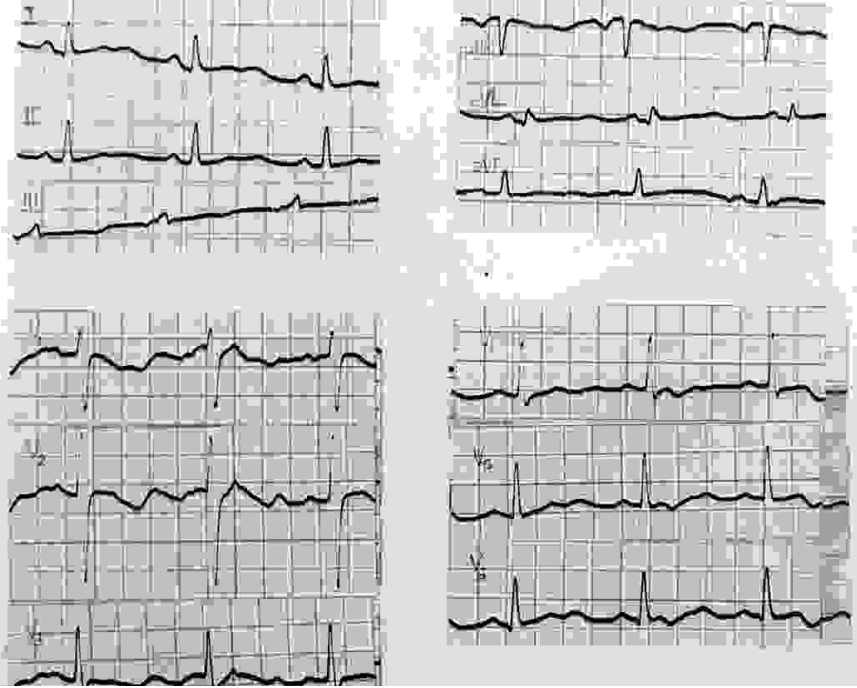

Fig. 1a. Fibrilación ventricular que revirtió tras choque de 200 Julios.

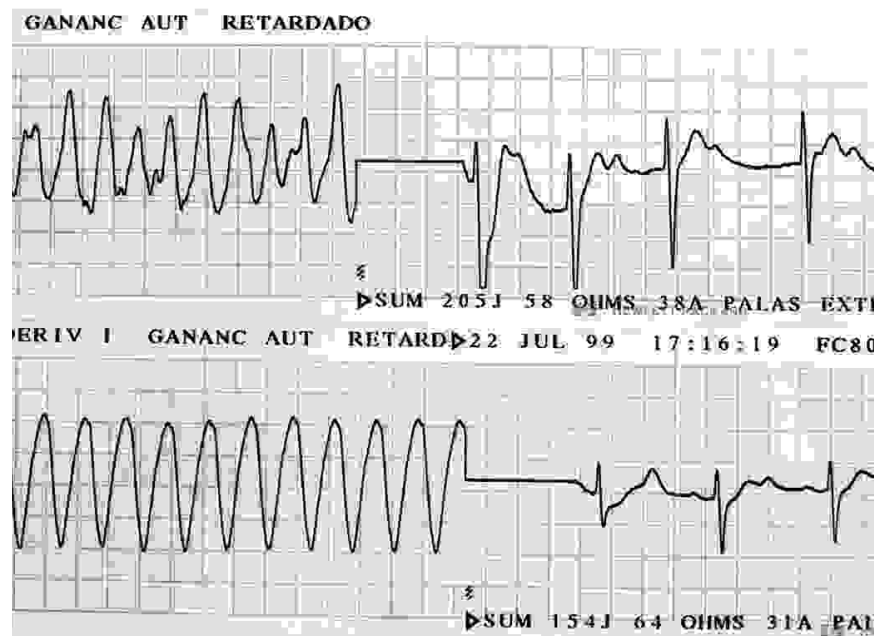

Fig. 1b. Flutter ventricular que revirtió a ritmo sinusal con choque de 150 Julios.

Las complicaciones cardiovasculares de la fenitoína intravenosa son más frecuentes cuando se administra a velocidad $>50$ $\mathrm{mg} / \mathrm{minuto}$, en mayores de 50 años y si existe enfermedad cardiaca previa. No obstante, también se han descrito en pacientes jóvenes y sanos como la nuestra, así como con velocidades de infusión $<50 \mathrm{mg} /$ minuto (2-5). La hipotensión representa un 28-50\% y las arritmias cardiacas un 2\% (5), siendo las más frecuentes los latidos ectópicos, la bradicardia y el bloqueo auriculoventricular completo (1). La evolución de estos pacientes es favorable tras suspender o disminuir la velocidad de infusión $(1,3,5)$, pero se han descrito casos mortales en pacientes con intoxicación digitálica $(6,8)$, enfermedad pulmonar severa (2), alteraciones metabólicas o anormalidades de la conducción ventricular (1).

Las complicaciones cardiovasculares de la fenitoína pueden ser debidas a la fenitoína en sí o a su diluyente, el propilenglicol $(2,5)$. Este último se ha sugerido produce estimulación vagal y efecto depresor miocárdico directo, cuyo resultado es hipotensión y arritmias cardíacas $(2,9)$. Los efectos electrofisiológicos de la fenitoína y la lidocaína son similares (10), pues ambos son antiarrítmicos clase IB. Las acciones que la fenitoína ejerce sobre las fibras atriales y de Purkinje dependen de su concentra- 
ción, la concentración de potasio extracelular y de las condiciones electrofisiológicas de las fibras cardíacas (8). Asi cuando las concentraciones de potasio y fenitoína son bajas puede aumentar el potencial de reposo de membrana, la velocidad máxima de fase 0 y la amplitud del potencial de acción de las fibras auriculares y de Purkinje; esto es mas pronunciado con exposición al frío, trauma mecánico o concentraciones tóxicas de digital. La eficacia de fenitoína en arritmias por toxicidad digitálica puede deberse a su acción depresora del simpático eferente. Los efectos de la fenitoína en los nodos sinoauricular y auriculoventricular (NAV) en el corazón in situ son variables, debiendo usarse con precaución en pacientes con NAV enfermo y en arritmias ventriculares en presencia de flutter o fibrilación auricular. La fenitoína no afecta significativamente a la conducción del sistema de His-Purkinje $(1,9,10)$, aunque debe usarse con cautela en pacientes con alteraciones de la conducción intraventricular o bloqueo de rama completo.

En conclusión, la administración de fenitoína intravenosa debe realizarse con monitorización electrocardiográfica aún en jóvenes sin patologia cardiovascular, ya que pueden producirse arritmias cardíacas graves como la fibrilación-flutter ventricular.

\section{E. Merlo González, F. Árbol Linde, J. Rodríguez*, P. López Onega}

Sección de Medicina Intensiva. *Servicio de Medicina Interna. Hospital "Nuestra Señora del Prado". Talavera de la Reina. Toledo

1. Randazzo DN7 Ciccone A, Schweitzer P, Winters SL. Complete atrioventricular block with ventricular asystole following infusion of intravenous phenytoin. J Electrocardiol 1995; 28: 157-9.

2. Hitotsumatsu T, Iwaki T, Fukui M, Tateishi J. Toxic myocardial damage due to intravenous phenytoin administration. Histopathology 1995; 26: 479-80.

3. Earnest MP, Marx JA, Drury LR. Complications of intravenous phenytoin for acute treatment of seizures. JAMA 1983; 249: 762-5.

4. Rall TW, Schleifer LS. Drogas efectivas para el tratamiento de la epilepsia. En: Goodman A, Goodman LS, Rall TW, Murad F, eds. Las bases farmacológicas de la terapéutica. Madrid: Medica Panamericana; $1986 \mathrm{p} 432-55$

5. Lowenstein DH, Alldredge BK. Status epilepticus. N Engl J Med 1998; 338: 970-6

6. Zoneraich S, Zoneraich O, Siegel J. Sudden death following intravenous sodium diphenylhydantoin. Am Heart J 1976; 91375-7.

7. Payne TA, Bleck TP. Status epilepticus. Crit Care Clin 1997; 13: 17-38.

8. Gellerman GL, Martínez Ch. Fatal ventricular fibrillation following intravenous sodium diphenylhydantoin therapy. JAMA 1967; 200:337-8

9. Wit AL, Rosen MR, Hoffman BF. Electrophysiology and pharmacology of cardiac arrhythmias. V1ll. Cardiac effect of diphenylhydantoin. Am Heart J 1975: 90: 397-404.

10. Bigger JT, HoffmanF. Drogas antiarrítmicas. En: Goodman A, Goodman LS, Rall TW, Murad F, eds. Las bases farmacológicas de la terapéutica. Medica Panamericana; Madrid, 1986; 713-46.

\section{Viagra y oftalmología}

\section{Sr. Director:}

El citrato de sildenafil (Viagra) tiene una indicación clara que es la disfunción eréctil $(1,2)$ como inhibidor selectivo de la fosfodiesterasa 5 (FDE-5) presente en la musculatura vascular de los cuerpos cavernosos del pene, mantiene los efectos del óxido nítrico prolongando la erección $(1,2)$.

Mucho se sabe de los efectos secundarios sistémicos (cefaleas, infartos) (2) pero poco se ha publicado en la bibliografía oftalmológica. Recientemente hemos visto un caso que tiene interés oftalmológico:
Paciente de 61 años con diabetes mellitus tipo 2 sin retinopatía y cardiopatía isquémica en tratamiento con beta-bloqueantes. Entre sus antecedentes oftalmológicos destaca el padecer retinosis pigmentaria no sindrómica con agudezas visuales máximas corregidas de 0,8 en ambos ojos. Utiliza viagra por prescripción urológica a una dosis de $50 \mathrm{mg}$. Refiere visión borrosa y en color azul brillante una hora después de la ingestión de $100 \mathrm{mg}$ (el doble de la indicada por su médico) y que duró 4 horas. No se produjo erección. Pasado el efecto la agudeza visual se recuperó a 0.8 y la sensibilidad al contraste y el campo visual no se modificaron respecto a pruebas anteriores.

El citrato de Sildenafil es una de las pastillas "milagro" aprobadas por la FDA (2) que actúa inhibiendo la FDE-5 en los cuerpos cavemosos, lo que produce un aumento del óxido nítrico y éste del GMPc que relaja la musculatura lisa aumentando el flujo sanguíneo y la erección durante horas. Este fármaco, en contra de lo que se pensaba, actúa sobre la retina (3) inhibiendo la FDE-6 esencialmente en los segmentos externos de los conos y bastones, lo que produce aumento de GMPc retiniano de forma prolongada dañando los fotoreceptores, por tanto viagra podría no estar indicada en los pacientes con retinitis pigmentosa sobre todo si tienen defectos en el gen de la fosfodiesterasa tipo 6 que produce retinitis pigmentosa. Al efecto tóxico sobre los fotoreceptores se podría sumar una congestión coroidea similar a la de los cuerpos cavernosos (1), con lo que se disminuiría el metabolismo de la porción externa de la retina acumulándose metabolitos que pueden incrementar la toxicidad.

Es importante que se conozcan bien los efectos secundarios de este fármaco por tres motivos: a) es utilizado frecuentemente; b) son pacientes que por su edad y por patologías vasculares asociadas que se traducen a otras partes del organismo acuden a diferentes especialistas y c) generalmente se sobredosifican sobrepasando las dosis óptimas recomendadas, y así cuando la ingesta es de $100 \mathrm{mg}$ se multiplica por cinco la toxicidad oftalmológica.

Por tanto consideramos que viagra debe ser utilizado con reserva en los pacientes en tratamiento con nitratos y debe ser administrada con precaución en las retinopatías pigmentosas y en todos los pacientes con antecedentes de procesos hipotensivos ya que puede potenciar neuritis ópticas y otros fenómenos isquémicos neuro-oftalmológicos.

\section{M. Asensio Sánchez}

Servicio de Oftalmología. Hospital del Insalud. Medina del Cam po (Valladolid).

1. Goldstein I, Lue TF, Padma-Nathan H, Rosen RC, Steers WD, Wicker PA. Oral sildenafil in the treatment of erectile dysfunction: Sildenafil Study Group. N Engl J Med 1998; 338:1397-404.

2. Center for Drug Evaluation and Research. Viagra (sildenafil). Joint Clinical Review for NDA-20-895. Washington, DC. Center for Drug Evaluation and Research, Food and Drug Administration; 1998.

3. Vobig MA, Klotz T, Staak M, Bartz-Schmidt KV, Engelmann U, Walter P. Retinal side-effects of sildenafil. Lancet 1999; 353: 375.

Exoftalmos unilateral secundario a metástasis orbitaria como manifestación inicial de un cáncer de mama

\section{Sr. Director:}

Por su rareza, presentamos el caso de una enferma con cáncer de mama cuya manifestación inicial fue un exoftalmos unilateral secundario a una metástasis única orbitaria. 